\title{
CRISPR standards
}

\author{
With the ever-expanding use of CRISPR technology, the development of standards to \\ quantitatively benchmark on- and off-target activity needs to keep pace.
}

Open almost any biological journal these days and there will be reports of CRISPR-based applications, from single-gene knockouts to gain- and loss-of-function screens to base editing or transcriptional regulation. Nature Methods is no exception, and this month's issue includes the use of CRISPR for genetic interaction mapping, a method to select edited cells without a marker, and two in vitro methods for Cas9 off-target determination.

Concerns about specificity have accompanied this technology from its beginnings. While a cell is well equipped to deal with the relatively random DNA damage induced by external stress, the breaks introduced by the guide RNA-Cas9 complex are not arbitrary and therefore are potentially more dangerous. They occur at sites with homology to the guide RNA (sgRNA) and can include off-target sites-loci other than the intended one. The Cas9 nuclease will repeatedly cut the same site until it is mutated, a desired outcome at the intended locus but not at off-target sites, particularly if the mutations are deleterious. Especially for applications aimed at use in the clinic, it is therefore critical to carefully assess specificity.

A Correspondence by Mahajan and colleagues (p547), highlights the need for such assessment by showing that the same sgRNA that repaired a mutation leading to blindness in mice also introduced a number of unanticipated mutations into the genome. Follow-up studies that seek to repeat this finding with different sgRNAs and to assess the deleteriousness of the off-target mutations will be of much interest.

However, to make it possible to productively compare CRISPR experiments across labs, more than just new assays to find off targets are needed. It will be essential to develop more standardized approaches to detect and quantify different editing outcomes, as well as report their level of confidence, so that the diverse editing tools that are rapidly becoming available can be compared for their activity and specificity.

DNA reference materials with known ground truth, in terms of sequence and structural variants, will be invaluable to assess where the main sources for variability in different methods lie. Physical DNA references will, for example, allow users to assess the quality of their measurement methods or to compare different off-target detection approaches, and will enable the optimization of methods for the detection of rare editing events. In silico standards will be valuable to determine the quality and comparability of current computational analysis pipelines and will also enable the development of muchneeded new computational tools, such as software for the detection of small insertions and deletions, to end the current practice of every lab developing their own tools to find Cas-induced indels.

Of course, the best-thought-out experiment will be of limited use to others if it is not accompanied by information about the data and the underlying methods. The development of guidelines for reporting the pertinent metadata that fully describe a CRISPR experiment will benefit the field in the same way that minimum information guidelines have done for other fields, for example the MIAME standard for microarray experiments. Such metadata should include the number of sgRNAs used, their sequence, and the tool used to design them. Controls should be specified, for example whether, and how many, scrambled or random sgRNAs were included and whether Cas9 alone was used. In addition, tools and methods for off-target assessment should be described.

Recent discussions between leaders in the genomeediting community and the US National Institute of Standards and Technology (NIST) have initiated the formation of a consortium that addresses these needs and consequently will develop measurement metrics, reference materials and recommendations for metadata reporting.

NIST has a successful track record when it comes to bringing together such consortia. As high-throughput sequencing emerged as a transformative technology over the last decade, the community identified the need to measure the quality of sequencing, assess error rates and judge the capabilities of software tools to analyze the data. NIST was instrumental in forming the 'Genome in a Bottle' consortium, a public-privateacademic enterprise to develop well-characterized, stable human reference materials, analysis methods and metrics to assess their performance. Ultimately the consortium hopes to create infrastructure that helps translate advances in genome sequencing to the clinic. The genome-editing consortium is still in the planning stages, but will be modeled after Genome in a Bottle and likewise aims to be open to all who want to participate.

We encourage CRISPR users to follow the development of the emerging consortium and to participate in the effort to create these reference materials and guidelines. 